\title{
TALOS: A new humanoid research platform targeted for industrial applications
}

\author{
O. Stasse ${ }^{1}$ and T. Flayols ${ }^{1}$ and R. Budhiraja ${ }^{1}$ and K. Giraud-Esclasse ${ }^{1}$ and \\ J. Carpentier ${ }^{1}$ and J. Mirabel ${ }^{1}$ and A. Del Prete ${ }^{1}$ and P. Souères ${ }^{1}$ and N. Mansard ${ }^{1}$ and \\ F. Lamiraux ${ }^{1}$ and J.-P. Laumond ${ }^{1}$ and L. Marchionni ${ }^{2}$ and H. Tome ${ }^{2}$ and F. Ferro ${ }^{2}$
}

\begin{abstract}
The upcoming generation of humanoid robots will have to be equipped with state-of-the-art technical features along with high industrial quality, but they should also offer the prospect of effective physical human interaction. In this paper we introduce a new humanoid robot capable of interacting with a human environment and targeting industrial applications. Limitations are outlined and used together with the feedback from the DARPA Robotics Challenge, and other teams leading the field in creating new humanoid robots. The resulting robot is able to handle weights of $6 \mathrm{~kg}$ with an out-stretched arm, and has powerful motors to carry out fast movements. Its kinematics have been specially designed for screwing and drilling motions. In order to make interaction with human operators possible, this robot is equipped with torque sensors to measure joint effort and high resolution encoders to measure both motor and joint positions.

The humanoid robotics field has reached a stage where robustness and repeatability is the next watershed. We believe that this robot has the potential to become a powerful tool for the research community to successfully navigate this turning point, as the humanoid robot HRP-2 was in its own time.
\end{abstract}

\section{INTRODUCTION}

The DARPA Robotics Challenge (DRC) was motivated by the design of several high performance humanoid robots such as ATLAS, or LOLA [1]. It has led to the design of new powerful robots such as Walkman [2], S-One/JAXON [3], PROXI/DURUS [4], [5] and the upgrade of standard platforms such as HRP-2 with HRP-2 Kai [6] or HUBO with DRC-HUBO+ [7]. Following the example of the initial DARPA Challenge on autonomous cars, it is interesting to investigate now the possible industrial outcome of the technologies developed for this competition and to integrate some of the hard lessons learned on this occasion. To realize such investigation, the difficulty is to find an available prototype of humanoid robot that integrates the recent technologies. This paper presents such a humanoid robot called Pyrène , depicted in Fig. 1, resulting from the collaboration of a robotics company and a research team.

\section{A. Availability of recent humanoid robot technologies}

Humanoid robots have to perform reactive motions to keep balance, which require actuators that can generate at the same time high torques and high speeds. We will quantify

\footnotetext{
*This work was supported by the ERC Grant ACTANTHROPE

1 LAAS-CNRS, Université de Toulouse, CNRS, Toulouse, France, firstname. familynamedlaas.fr

$\begin{array}{lll}2 & \text { PAL } & \text { Robotics, Bain } \\ \text { fistname.familynameapal-robotics.coma, } & \end{array}$
}

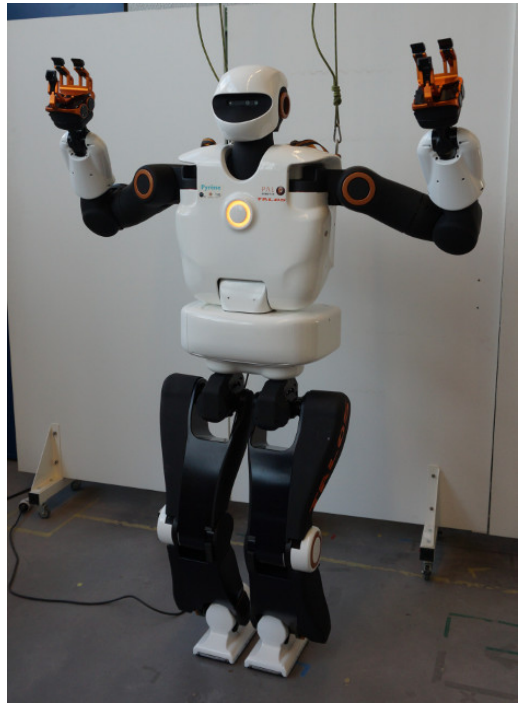

Fig. 1. Pyrène the first robot of the TALOS serie built by PAL-Robotics.

clearly these requirements for possible industrial applications through specific examples in the paper. According to Englsberger and al. [8], the Atlas robot provides high torques with high bandwidth, but it is generating loud sounds, needs high power and has high friction/stiction that complexify its control. In addition, discussions with industrial partners pointed out that hydraulic systems have less chance to be certified than electrical motor based robots due to their high pressure, and the difficulty to implement the needed security features.

On the other hand the seminal work of Urata [9], leading to the design of the S-one robot from Schaft, shows that it is possible to realize powerful motions with electric motors. WALK-MAN [2] and DRC-HUBO+ [7] are robots using this kind of technology. WALK-MAN has new high performance actuators with impressive capabilities, but it is a laboratory prototype and the maintenance and support are difficult to perform outside IIT. The DRC-HUBO+ (leg joints) as well as the HRP-2 Kai (pitch joints of the legs) have two motors in parallel, which are increasing the torque capabilities but not the speed. In addition the DRC-HUBO+ is using CAN bus, which is a bottleneck for high-frequency access to the low-level controllers (e.g. $1 \mathrm{Khz}$ ). To mitigate this problem, recent robots use EtherCAT (e.g. HRP-2 Kai and WALK- 
MAN) for their communication bus.

Humanoid robots such as Valkyrie [10] or WALK-MAN [2] use Serial Elastic Actuators (SEA). SEA can absorb impacts, store the related energy and release it later. In addition, the spring can be used to measure the joint torque. However, SEA makes the robot control more complex as the actuator dynamics needs to be taken into account [11].

Torque controlled robots such as TORO [8] or DURUS [5] are very promising to deal with unplanned contact with the environment and humans. Unfortunately TORO is unlikely to be available outside DLR. Recent robots such as REEM-C have encoders both at the motor and at the joint side. Provided that the encoder resolution at the joint side is sufficient, it is possible to use them to model the deflection induced by the harmonic drive. This deflection is proportional to the torque.

DURUS is another new humanoid robot sold by SRI with impressive efficiency capacities with springs at the ankles and torque control. The transmission has been designed to have low friction, and the robot has torque sensing in all the joints. However, even if the spring constrained along the vertical axis at the ankles is efficient to store energy and absorb impacts, its implication during manipulation is less clear. Our experience on several applications is that humanoid robots are usually used in acyclic behaviors, and mix high-stiffness (for manipulation and support legs) with low-stiffness (for impacts and human-robot interaction). This stiffness regulation can be provided either by mechanical design or by control. A mechanical compliance can be particularly useful during landing to filter impact. However, during high-precision manipulation the mechanical compliance needs to be controlled, and most of the current solutions are not able to provide the same stiffness of a rigid system. Therefore the approach for Pyrène was to have a controlled compliance, and not a mechanical one.

As torque control is still a research topic, Pyrène allows for both position and torque control.

Finally the recent advances on whole-body control rely mostly on complex optimization problems solved in an efficient manner [12], [13], [14], [15], [16]. This involves a significant computational power available on the robot.

\section{B. Applications}

In this paper, we focus mostly on partial scenarios coming from Aircraft manufacturing where humanoid robots have a potential interest over other mobile platforms, such as climbing stair cases, or going through narrow passages. After presenting our current results on the humanoid robot HRP-2, dynamical simulations on the new robot are presented.

Another point that drove the robot specifications was musculo-skeletal stress in human operators. Operations in aircraft manufacturing imply frequent passages in narrow spaces while handling heavy tools with stretched arms. This imposes to have a robot able to handle tools weighting up to $6 \mathrm{~kg}$ with stretched arms. The environments in which the robot has to evolve include narrow spaces and stairs, similar to the ones found in the DRC. Although robots with

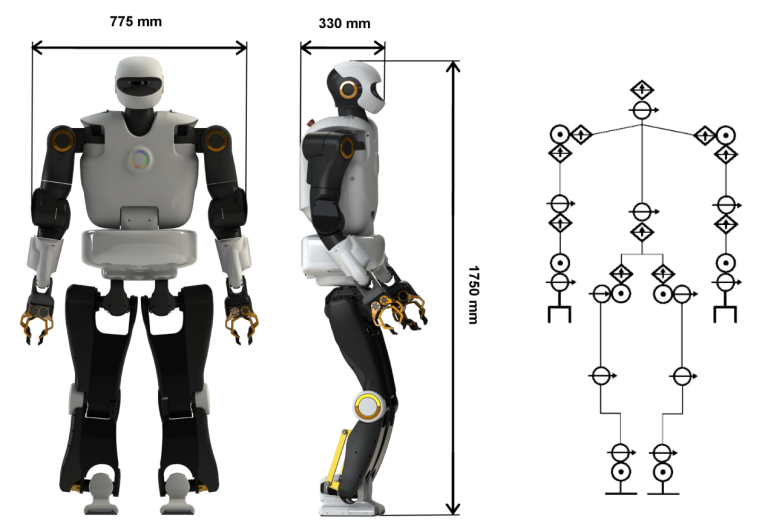

Fig. 2. Kinematics of TALOS.

\begin{tabular}{|c|c|c|c|c|}
\hline & HRP-2 Kai & WALK-MAN & Pyrène & DRC-HUBO+ \\
\hline Height & $1.710[\mathrm{~mm}]$ & $1915[\mathrm{~mm}]$ & $1750[\mathrm{~mm}]$ & $1750[\mathrm{~mm}]$ \\
\hline Width & $629[\mathrm{~mm}]$ & $\begin{array}{l}815[\mathrm{~mm}] \\
600[\mathrm{~mm}]\end{array}$ & $775 \mathrm{~mm}-550[\mathrm{~mm}]$ & $540[\mathrm{~mm}]$ \\
\hline Weight & $65 \mathrm{~kg}$ & $132 \mathrm{~kg}$ & $95 \mathrm{~kg}$ & $80 \mathrm{~kg}$ \\
\hline Total DoFs & 32 & 33 & 32 & 30 \\
\hline Head & 2 D.O.F. & 2 D.O.F. & 2 D.O.F. & 1 D.O.F. \\
\hline Arm & $2 \times 7$ D.O.F. & $2 \times 7$ D.O.F. & $2 \times 7$ D.O.F. & $2 \times 7$ D.O.F. \\
\hline Hand & $2 \times 1$ D.O.F. & $2 \times 1$ D.O.F. & $2 \times 1$ D.O.F. & $2 \times 1$ D.O.F. \\
\hline $\begin{array}{l}\text { Waist } \\
\end{array}$ & 2 D.O.F. & 3 D.O.F & 2 D.O.F. & $\begin{array}{l}1 \text { D.O.F. } \\
\times 6 \text { DDOF }\end{array}$ \\
\hline & & & & \\
\hline
\end{tabular}

TABLE I

OVERALL DESCRIPTION OF ROBOTS USED IN THE DRC

wheels have already been investigated in Aircraft Industry, they involve the need of elevators and thus increase the cost of deployment.

As well as in the context of research, an important factor for the deployment of humanoid robots in real scenario is the repeatability. This goal can be reached when the design of the robot is done in collaboration with an industrial partner mastering the integration known-how, or with institutes having strong technological centers such as DLR [8] and IIT [2]. The successful production of the HRP series [17], [18], and the robots from Boston Dynamics are good examples of robots with industrial quality. For this reason Pyrène, the robot presented in this paper, was built by the company PALRobotics. PAL-Robotics has also successfully realized the REEM-B [19] and REEM-C humanoid robots. Pyrène is the first prototype of the TALOS series. The robot was specified by the Gepetto team of the LAAS-CNRS laboratory, based upon its expertise in humanoid robots.

\section{SPECIFICATIONS OF PYRÈNE}

\section{A. Kinematics constraints}

The kinematics of Pyrène are depicted in Fig. 2

1) Range and overall structure: Pyrène has almost the same joint range as the average human model[20] (see Table III). Its range of motion is wider than ATLAS, except for the ankle inversion. Conversely it has a slightly smaller range than WALK-MAN for almost all joints. It has 32 DoFs as most recent humanoid robots (see Table [I-A), such as HRP-2 Kai, WALK-MAN, and DRC-HUBO+. In terms of weight, with $95 \mathrm{~kg}$ Pyrène is heavier than DRC-HUBO+ and HRP-2 Kai, but lighter than WALK-MAN and ATLAS. 

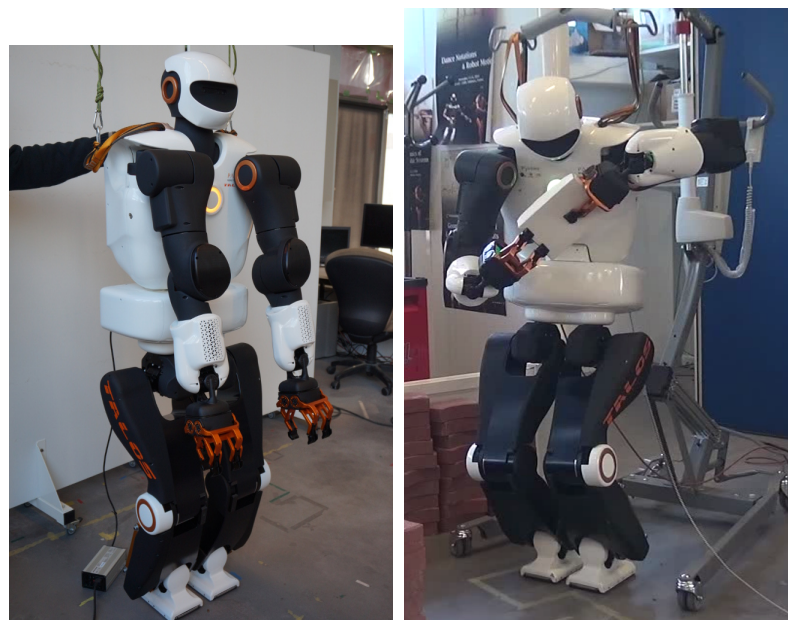

Fig. 3. (left) Pyrène folding its arms in front. (right) Planning a manipulation behavior

\begin{tabular}{|c|c|c|c|c|}
\hline Joint & Human body & WALK-MAN & TALOS & ATLAS \\
\hline Hip pitch & $-120.0,+10.0$ & $-120.0,+60.0$ & $-120.0,+45.0$ & $-92.38,+37.68$ \\
Hip roll & $-40.0,+30.0$ & $-50.0,+40.0$ & $-30.0,+30.0$ & $-30.0,+30.0$ \\
Hip yaw & $-35.0,+35.0$ & $-90.0,+50.0$ & $-20.0,+90.0$ & $-10.0,+45.0$ \\
Knee & $0.0,+145.0$ & $0.0,+140.0$ & $0.0,+150.0$ & $0.0,+135.0$ \\
Ankle pitch & $-40.0,+15.0$ & $-80.0,+60.0$ & $-75.0,+45.0$ & $-57.0,+40.0$ \\
Ankle roll & $-30.0,+30.0$ & $-45.0,+45.0$ & $-30.0,+30.0$ & $-45.0,+45.0$ \\
\hline
\end{tabular}

TABLE II

RANGE OF THE LEG JOINTS OF DIFFERENT ROBOTS.

2) Shoulders: In contrast to HRP-2 and HRP-2 Kai, Pyrène has been designed to have a maximum manipulability in its front in order to perform drilling and screwing motions. For this reason, the first axis of the shoulder, instead of being along the pitch axis, is along the yaw axis. In this way, when both shoulders are folded in the front (see Fig. 3-left) the robot has a width of $550 \mathrm{~mm}$ instead of $775 \mathrm{~mm}$. It becomes then more narrow than HRP-2 Kai. In addition, HRP-2 has only 6 DoFs in its arms, thus no redundancy to control the $6 \mathrm{~d}$ pose of the end-effector. While this simplifies the computation of the inverse kinematics, it severely limits the manipulability if additional constraints need to be handled. The generalization of numerical methods to deal with the problem of redundancy makes now the interest of analytical solution weaker.

3) Hip and knee: From the kinematic viewpoint the cantilever structure of HRP-2 is interesting to alternatively put one foot in front of the other when going through narrow spaces. However, this structure is not ideal for legs with higher-powered motors because it puts more stress on the mechanical structure and increases the width of the robot. For these reasons, the two legs, although close to each other, are designed not to collide while spanning a wide range of motion.

\section{B. Batteries}

Pyrène is equipped with Li-CNM (Cobalt-NickelManganese) batteries, which are able to deliver $74 \mathrm{~V} D C$ with a capacity of $15 A h$. Compared to HRP-2 Kai the

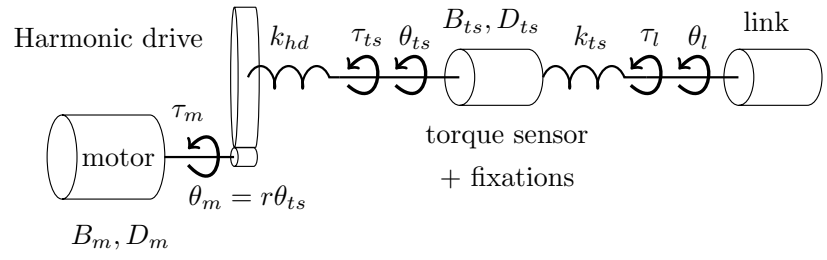

Fig. 4. Structure of Pyrène actuator.

\begin{tabular}{|c|c|}
\hline$k_{t s} \approx 270 \mathrm{KNm} / \mathrm{rad}$ & Torque sensor stiffness \\
$k_{h d} \approx 540 \mathrm{KNm} / \mathrm{rad}$ & HD stiffness \\
$B_{t s}=0.033 \mathrm{kgm}$ & Inertia of the torque sensor \\
$B_{m}$ & Motor inertia \\
$D_{t s}=0$ & Damping of the torque sensor \\
$D_{m}=0$ & Motor Damping \\
$T_{f r}$ & Friction of the HD \\
$K_{m}=0.1$ & Motor torque constant \\
$r=100$ & HD reduction ratio \\
$\theta_{t s}$ & Position of the torque sensor \\
$\theta_{m}$ & Position of the motor \\
$\tau_{l}$ & Load torque \\
$R=200 \mathrm{~m} \Omega$ & Armature resistance \\
$V$ & Armature Voltage \\
$K_{b}=80 \mathrm{rpm} / \mathrm{V}$ & Back-EMF constant \\
$i_{a}$ & motor current \\
\hline
\end{tabular}

TABLE III

LIST OF ACTUATOR PARAMETERS.

capabilities of the batteries are $50 \%$ higher. Finally, if powerful motions are needed, the batteries are also able to deliver peaks of $150 \mathrm{~A}$. These capabilities are driven by the work of Urata, which led to the S-One robot [9].

\section{Actuators}

The structure of Pyrène actuators is depicted in Fig. 4 A brushless motor is connected to a Harmonic Drive (HD), which is itself connected to a torque sensor. Finally the torque sensor is connected to a link. Two high-precision encoders (19 bits) measure the motor and joint positions, $\theta_{m}$ and $\theta_{l}$ respectively. This relationship gives rise to the following set of equations linking the motion and the motor parameters:

$$
\begin{aligned}
& k_{t s}\left(\theta_{t s}-\theta_{l}\right)=\tau_{l} \\
& B_{t s} \ddot{\theta}_{t s}+D_{t s} \dot{\theta}_{t s}+k_{h d}\left(\theta_{l}-\frac{\theta_{m}}{r}\right)=\tau_{t s} \\
& T_{f r}\left(\tau_{m}, \tau_{l}, \theta_{m}\right)+B_{m} \ddot{\theta}_{m}+D_{m} \dot{\theta}_{m}-k_{h d}\left(\frac{\theta_{m}}{r}-\theta_{t s}\right) \\
& =K_{m} i_{a} \\
& \frac{V}{R}-\frac{K_{b}}{R} \dot{\theta}_{m}=i_{a} \\
& K_{m} i_{a}=\tau_{m}
\end{aligned}
$$

where the parameter definitions are given in Table III

This model is very similar to the one found in [21]. In practice we found out on HRP-2 that most of the problems arised from: power limitation $\left(P=V i_{a}\right)$, temperature overheating, limitation in torques from the motor $\left(\tau_{m}\right)$ or the HD $\left(\tau_{t s}\right)$. The maximum peak motor torque given by the data sheet is usually higher than the maximum peak torque for the HD. In addition the peak motor and HD torques can 
be exceeded during a short time interval. For instance, the biggest HD available in WALKMAN is a CPL series, size 25 , ratio 80 (according to [22]). According to the datasheet, the momentary peak torque is $255 \mathrm{Nm}$. This corresponds to the kind of peak torque we have experimented on HRP-2 for high-demanding motions during $10 \mathrm{~ms}$, despite the lower limits of this robot 1

It is more difficult to take into account the torque limits induced by a large torque applied over a longer period of time, or repeatedly. This creates internal temperature builtup. Therefore the torque and the time during which one can apply it depend on the environment temperature and the past usage of the motor. It is possible to create a model of the motor temperature behavior [23]. The HDs are also subject to constraints related to the application, indeed a repeatable peak torque (cycling walking for instance) is lower than momentary peak torque (unique explosive motion). For instance, in the case of the HD example used previously, the repeatable peak torque is $137 \mathrm{Nm}$.

As depicted in Table IV, Pyrène seems to have lower power and speed than WALKMAN according to [2]. However, looking at [22], which gives a more thorough description of the actuator, one can remark that the numbers given in [2] do not consider the torque limits of the HD. For this reason three values are given for the maximum torques of Pyrène in Table IV, each considering different factors: max motor torque, max motor drive current, and max HD momentary peak torque. The third column gives numbers higher than WALK-MAN [2], but the first column is similar to the numbers given in [22] where the HD limitations are considered.

Finally, this actuator allows for both position and torque control.

\section{Sensors}

1) IMU and force sensors: As common, Pyrène is equipped with an IMU at the level of its waist. This allows measuring the trunk orientation with respect to the gravity field and the robot global acceleration. In addition, Pyrène is equipped with 6-axis force/torque sensors at the level of the hands and the feet. The main difference with respect to HRP2 is the capacity of these sensors to sustain up to 6 times the weight of the robot, whereas it is 2 times on HRP-2.

2) Torque sensors: As mentioned in Section II-C, Pyrène is equipped with torque sensors in almost all the joints, except the two wrist DoFs and the two head DoFs. They directly measure the torque applied on the load side. We believe that the redundancy of the sensors, as well as their fast update due to the EtherCAT bus, are a key ingredient to achieve torque control on humanoid robots.

3) Vision: Pyrène is equipped with an ORBBEC Astra Pro RGB-D camera. The stairs used as a testbed for developing our algorithms are depicted in Fig. 6. The camera providing the RGB part is a CMOS camera using a rolling

\footnotetext{
${ }^{1}$ We cannot provide the exact limits of the HRP-2 robot due to a NonDisclosure Agreement.
}

shutter system, which can be problematic with dynamic scenes. The head has been designed to be modified, and we plan to add a stereoscopic system, or at least a CCD camera, together with an IMU for latter investigation. Fallon has shown in [24] that an advanced stereoscopic system can be highly reliable.

\section{E. Computational power}

The robot is supplied with two computers, each equipped with dual i7 CPU at $2.8 \mathrm{GHz}$. Each CPU has two cores and is hyper-threaded, which gives a total of 8 cores per computer. However, since the real-operating system used is RT-PREEMPT, only 4 cores are available on the control computer Eight cores are anyway available on the computer for vision and high-level computation. The motherboards are in a specific box called the logic box, which can be easily removed. This ensures proper cooling of the CPUs and facilitates their upgrade, a key factor for HRP-2 extended lifespan.

\section{F. Software environment}

PAL-Robotics has a history of providing its robots (REEM-C and TIAGO) with ROS deeply integrated. The operating system used in Pyrène is an Ubuntu 14.04 LTS. The robot low-level system is developed with ros_control. The robot can be simulated with Gazebo, and the multimedia system is using the stacks providing navigation and mapbuilding.

1) roscontrol: ros_control is an abstract interface that allows to switch easily from simulation to real tests on the robot. Applying this to Pyrène is not direct. Indeed the robot has additional joint state information that needs an extension of the interface. Therefore the robot is now working on a modified version of the mainstream ros_control and we hope to submit this modification in order to keep the compatibility with the system. More precisely, according to the model presented in Eq. 1. we need an interface to measure the joint position at the load side $\theta_{l}$ (not provided by ros_control) and the motor side $\theta_{m}$ (provided by ros_control). We also need the motor current $i_{a}$ (provided by ros_control) and the torque sensor $\tau_{t s}$ (not provided by ros_control). Thanks to ros_control we were able to port our software control architecture (Stack Of Tasks [25]) on Pyrène in less than a month.

ros_control is easier to use than the OpenRTM architecture, however it currently lacks the composability offered by OpenRTM [26]. For instance, on HRP-2, the stabilizer, the walking pattern generator and a dynamical library were provided as basic software bricks through OpenRTC components.

2) Simulation: Several simulators are available under ROS, but not all of them are compatible with ros_control. Gazebo is the simulator widely used in the robotics community as it is a system simulator that allows testing a large software framework. However, Gazebo is mostly relying on the ODE dynamic engine. Even if recent developments considerably improved the reliability of this engine, it still 


\begin{tabular}{|c|c|c|c|c|c|c|}
\hline & \multicolumn{2}{|c|}{ WALK-MAN } & \multicolumn{2}{|l|}{ TALOS } & \multicolumn{2}{|c|}{ Atlas } \\
\hline Joint & Max Torque & Max Speed & Max Torque & Max Speed & Max Torque & Max Speed \\
\hline Hip flex./extension/pitch & $400 \mathrm{Nm}$ & $9 \mathrm{rad} / \mathrm{s}$ & $157 \mathrm{Nm} / 284 \mathrm{Nm} / 400 \mathrm{Nm}$ & $8.58 \mathrm{rad} / \mathrm{s}$ & $260 \mathrm{Nm}$ & $12 \mathrm{rad} / \mathrm{s}$ \\
\hline Hip abduction/adduction/roll & $400 \mathrm{Nm}$ & $8.2 \mathrm{rad} / \mathrm{s}$ & $157 \mathrm{Nm} / 284 \mathrm{Nm} / 404 \mathrm{Nm}$ & $8.58 \mathrm{rad} / \mathrm{s}$ & $90 \mathrm{Nm}$ & $12 \mathrm{rad} / \mathrm{s}$ \\
\hline Hip rotation/yaw & $140 \mathrm{Nm}$ & $19.5 \mathrm{rad} / \mathrm{s}$ & $82 \mathrm{Nm} / 147 \mathrm{Nm} / 210 \mathrm{Nm}$ & $5.86 \mathrm{rad} / \mathrm{s}$ & $110 \mathrm{Nm}$ & $12 \mathrm{rad} / \mathrm{s}$ \\
\hline Knee & $400 \mathrm{Nm}$ & $16.2 \mathrm{rad} / \mathrm{s}$ & $308 \mathrm{Nm} / 465 \mathrm{Nm} / 648 \mathrm{Nm}$ & $8.58 \mathrm{rad} / \mathrm{s}$ & $890 \mathrm{Nm}$ & $12 \mathrm{rad} / \mathrm{s}$ \\
\hline Ankle plantar/dorsi flexion/pitch & $330 \mathrm{Nm}$ & $18.6 \mathrm{rad} / \mathrm{s}$ & $157 \mathrm{Nm} / 284 \mathrm{Nm} / 400 \mathrm{Nm}$ & $8.58 \mathrm{rad} / \mathrm{s}$ & $220 \mathrm{Nm}$ & $12 \mathrm{rad} / \mathrm{s}$ \\
\hline Ankle inversion/eversion/roll & $210 \mathrm{Nm}$ & $16.7 \mathrm{rad} / \mathrm{s}$ & $82 \mathrm{Nm} / 147 \mathrm{Nm} / 232 \mathrm{Nm}$ & $8.58 \mathrm{rad} / \mathrm{s}$ & $90 \mathrm{Nm}$ & $12 \mathrm{rad} / \mathrm{s}$ \\
\hline
\end{tabular}

TABLE IV

TORQUE AND SPEED RANGES FOR WALK-MAX [2], TALOS AND ATLAS. THE THREE VALUES FOR THE MAX TORQUES OF PYRÈNE CONSIDER: I) ALL THE LIMITS INCLUDING THE HD, II) THE MAX CURRENT OF THE MOTOR DRIVES AND THE MAX MOMENTARY PEAK TORQUE OF THE HD, III) ONLY THE MOTOR LIMITS.

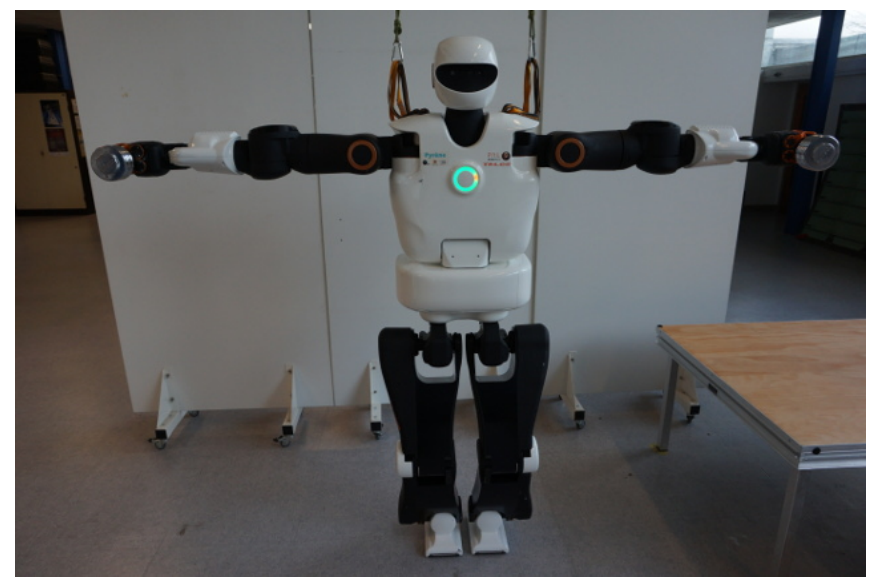

Fig. 5. Pyrène is holding two weights of $6 \mathrm{kgs}$ in each gripper with strechted arms.

suffers from problems to simulate biped robot walking. In our experience, OpenHRP/AIST dynamical engine provides a better result because the rigidity of the mechanical structure is guaranteed by the use of dedicated algorithms considering the kinematic tree of the robot. When the dynamics of the robot is computed, it is then included in a global dynamical system with generalized coordinates and contact-force detection. Regularization is achieved indirectly by introducing spring joints only in the parts of the system that are flexible. For instance, in HRP-2, there is a bush-rubber between the robot ankles and soils. Using this approach, the OpenHRP simulator together with HRP-2 has constantly provided us with meaningful simulations. We are currently working on a version tailored to the Pyrène robot. In the meantime, for motions that are not highly dynamics (e.g. moving slowly while standing), the dynamic simulation provided by Gazebo/ODE is sufficient.

\section{Simulations AND EXPERIMENTS}

In this section, the robot has been controlled using position control. The implementation of torque control is left for future work.

\section{A. Experiments}

1) Stretched arms: We have performed tests where the robot is holding a weight of $6 \mathrm{~kg}$ with each gripper with stretched arms, as depicted in Fig. 5. The actuator along the

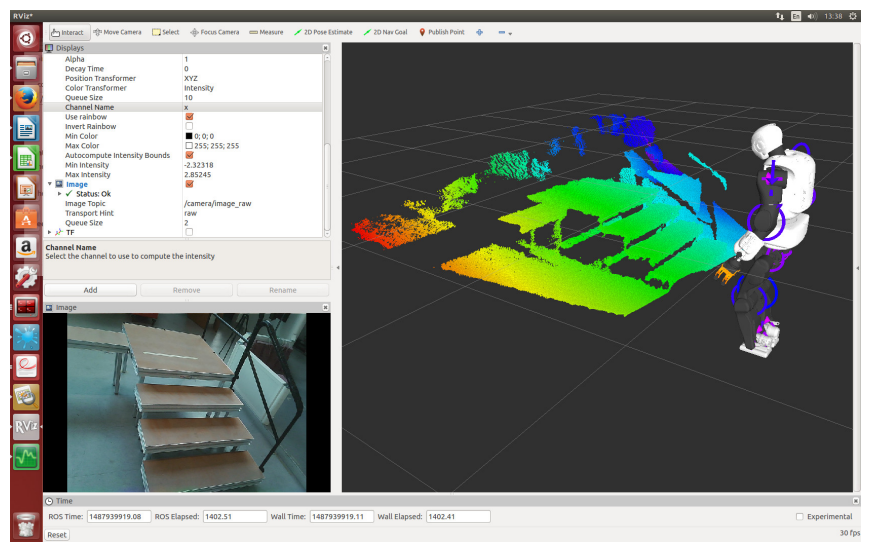

Fig. 6. View of the robot image and its RGB-D camera. The values of its torque sensors are also displayed

shoulder roll axis reached $75 \%$ of its maximum torque, with a slight bending at the shoulder and at the wrist.

2) Walking: Pyrène is supplied with the same walking pattern generator used for REEM-C. Fig. 7 depicts the robot walking with bricks in its hand. We kindly invite the interested reader to go at the following url to have a demonstration of the robot capabilities: https://youtu. be/SxdNvP2jKCC and to have a look to the companion video. The concept of this walking pattern generator is very similar to the one proposed in [27]. It does not use a dynamical model to filter the discrepancy between the inverted pendulum model and the momenta generated by the limbs during the motion. However, because it provides a fast analytical solution, the system is able to modify online its foot-steps according to the feedback of the CoP computed from the force-torque sensors available on both feet ${ }^{2}$ The walking is therefore very reactive and can be used with a joystick. We plan to improve this algorithm by using a method coupling dynamical filter with automatic choice of foot-steps [28]. This will be done after implementing another stabilizer on the robot.

The current stabilizer, included in the walking pattern generator provided by PAL-Robotics, is considering the effects of all the structural compliances by estimating the trunk orientation with respect to the gravity field. Pyrène has

\footnotetext{
${ }^{2}$ Several other methods using analytical solutions have been proposed in the past decade, due to space constraints we cannot list them all here
} 


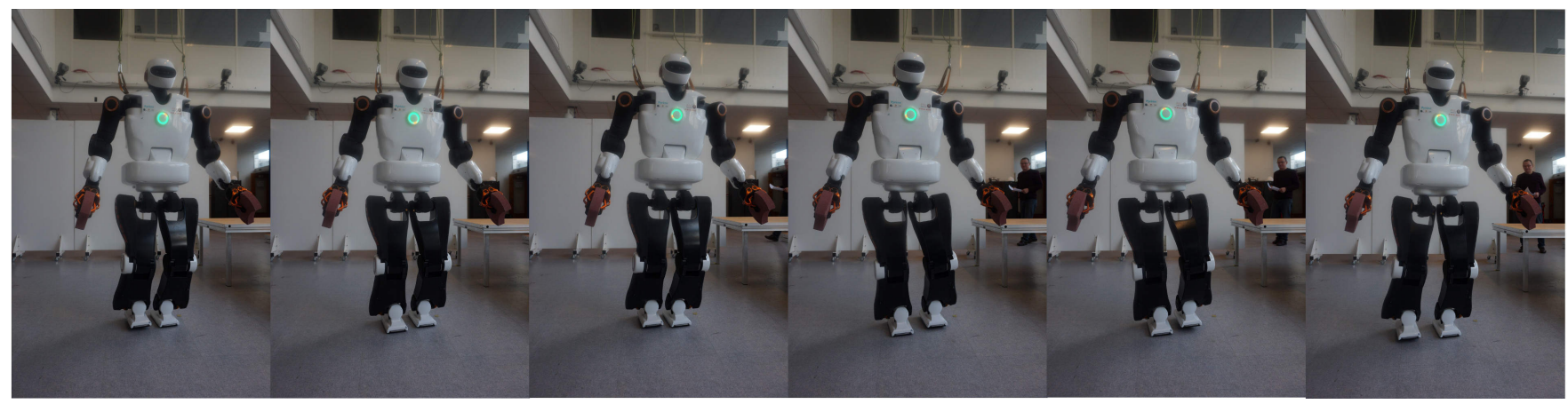

Fig. 7. Pyrène walking with bricks in both grippers. Each brick weight $1.5 \mathrm{~kg}$

structural compliances at the level of the hip, which are higher than the ones on HRP-2. However, compared to the HRP-2 compliance located at the ankles, the effect of this structural compliance on the CoP position is weaker. The disadvantage of not having a mechanical compliance to absorb impacts is the need to control accurately the robot dynamics. Our experience in tackling this issue is to have a walking pattern generator handling the robot dynamics. The strategy is to use it together with torque control to improve the walking performance.

\section{B. Climbing stairs}

1) Simulation: In a previous work [15] the Gepetto Team has proposed an algorithm to plan the centroidal dynamics for a given set of contact points in the stairs. In a second phase an Operational Space Inverse Dynamics is used to compute a dynamically consistent joint trajectory. This algorithm has been successfully applied to HRP-2. We used the same algorithm on the URDF model of Pyrène. The results in simulation are depicted in Fig. 8

2) Experiment: In [15] the motion played on the robot had no feedback control because the motion on the robot was consistent with the simulation. As Pyrène is heavier, this motion highlighted two structural compliances on the robot. One at the wrist, and the other at the level of the hip. Still, despite its weight, with a slight help the robot was able to climb the first stair. We believe that a torque control strategy should be able to compensate for this problem, and this is currently our main target. The experiment is depicted in the companion video.

\section{Motion planning}

Using the motion planner called HPP described in [29] we tried to plan an action involving manipulation. The robot starts with a position where it is holding a box in the left hand, and should finish with the box in the right hand. In HPP, the robot affordance is described for the hands and the feet. The box affordance is also given to the planner. From this, the planner is able to generate a motion which is taking into account quasi-static balance and the affordances (cf Fig. 3-right). The algorithm used to plan this motion is not taking into account the dynamical effects. Despite this, the robot was able to play the motion without jeopardizing the robot balance. The experiment is depicted in the companion video.

\section{CONCLUSION}

In this paper we have presented a new humanoid robot with an actuation technology using electric motors. This humanoid tries to take the best in the field in terms of communication buses, motor size, reduction, kinematics and highly redundant sensing of the actuators for tackling industrial applications.

\section{APPENDIX}

The participation of the authors to the work described in this paper is the following: O. Stasse (Gepetto TEAM Coordinator), T. Flayols (Gepetto TEAM - Electrotechnics specialist) , R. Budhiraja (Gepetto TEAM - Multi-contact and tests on Pyrène ), K. Giraud-Esclasse (Gepetto TEAM - Mechanical specialist and tests on Pyrène ), J. Carpentier (Gepetto TEAM - Multi-contact on Pyrène ), J. Mirabel (Gepetto TEAM - Planning on Pyrène ), A. Del Prete (Gepetto TEAM - Torque control on Pyrène ), P. Souères (Gepetto TEAM - Team leader) , N. Mansard (Gepetto TEAM - Motion control) , F. Lamiraux (Gepetto TEAM - Motion Planning), J.-P. Laumond (Gepetto TEAM Project Actanthrope leader), L. Marchionni (PAL Robotics Mechatronics specialist) , H. Tome (PAL Robotics - Software and Motion Control), F. Ferro (PAL Robotics - CEO )

\section{ACKNOWLEDGMENT}

This work was partially supported by the ERC-ADG 340050 Actanthrope, Project Flag-Era JTC 2016 RoboCom++ and the CNRS Institute INSII.

\section{REFERENCES}

[1] S. Lohmeier, T. Buschmann, and H. Ulbrich, "Humanoid robot lola," in IEEE/RAS Int. Conf. on Robotics and Automation (ICRA), 2009.

[2] F. Negrello, M. Garabini, M. G. Catalano, P. Kryczka, W. Choi, D. G. Caldwell, A. Bicchi, and N. G. Tsagarakis, "Walk-man humanoid lower body design optimization for enhanced physical performance," in IEEE/RAS Int. Conf. on Robotics and Automation (ICRA), 2016, pp. 1817-1824

[3] K. Kojima, T. Karasawa, T. Kozuki, E. Kuroiwa, S. Yukizaki, S. Iwaishi, T. Ishikawa, R. Koyama, S. Noda, F. Sugai, S. Nozawa, Y. Kakiuchi, K. Okada, and M. Inaba, "Development of life-sized highpower humanoid robot JAXON for real-world use," in IEEE/RAS Int Conf. on Humanoid Robotics (ICHR), 2015. 


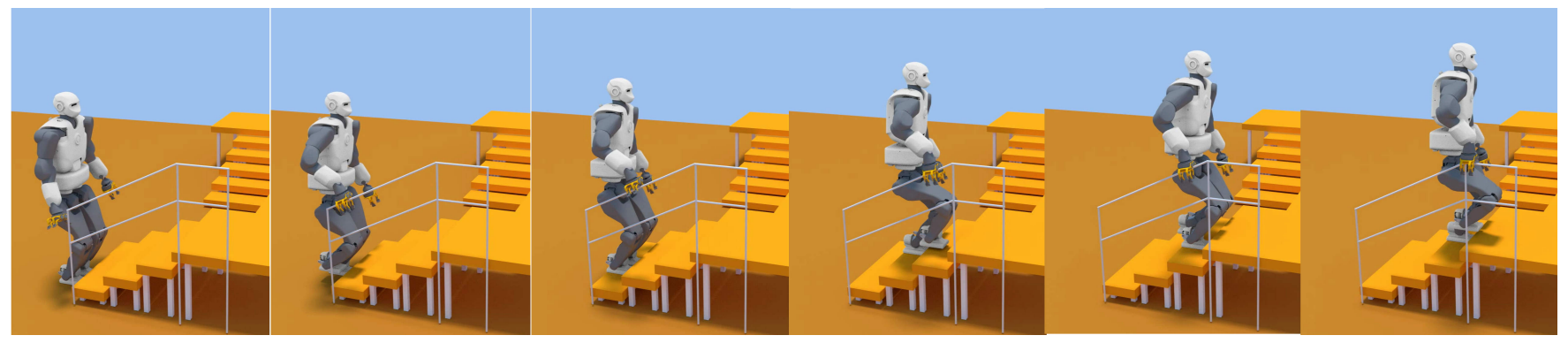

Fig. 8. Dynamical simulation of Pyrène climbing up stairs

[4] S. R. Institute, "Proxi: Redefining humanoid efficiency," 2016. [Online]. Available: https://www.sri.com/sites/default/files/brochures/ proxi-humanoid-robot.pdf

[5] A. Hereid, E. A. Cousineau, C. M. Hubicki, and A. D. Ames, "3d dynamic walking with underactuated humanoid robots: A direct collocation framework for optimizing hybrid zero dynamics," in IEEE/RAS Int. Conf. on Robotics and Automation (ICRA), 2016.

[6] K. Kaneko, M. Morisawa, S. Kajita, S. Nakaoka, T. Sakaguchi, R. Cisneros, and F. Kanehiro, "Humanoid robot hrp-2 kai - improvement of hrp-2 towards disaster response tasks," in IEEE/RAS Int. Conf. on Humanoid Robotics (ICHR), 2015, pp. 132-139.

[7] Rainbow, "http://www.rainbow-robotics.com/," January 2017.

[8] J. Englsberger, A. Werner, C. Ott, B. Henze, M. A. Roa, G. Garofalo, R. Burger, A. Beyer, O. Eiberger, K. Schmid, and A. AlbuSchffer, "Overview of the torque-controlled humanoid robot toro," in IEEE/RAS Int. Conf. on Humanoid Robotics (ICHR), 2014.

[9] J. Urata, Y. Nakanishi, and K. O. et M. Inaba, "Design of high torque and high speed leg module for high power humanoid," in IEEE/RSJ Int. Conf. on Intelligent Robots and Systems (IROS), 2010.

[10] N. A. Radford, P. Strawser, K. Hambuchen, J. S. Mehling, W. K Verdeyen, A. S. Donnan, and al., "Valkyrie: Nasa's first bipedal humanoid robot," Journal of Field Robotics, vol. 32, no. 3, pp. 397419, 2015

[11] N. Paine, J. S. Mehling, J. Holley, N. A. Radford, G. Johnson, C.L. Fok, and L. Sentis, "Actuator control for the nasa-jsc valkyrie humanoid robot: A decoupled dynamics approach for torque control of series elastic robots," Journal of Field Robotics, vol. 32, no. 3, pp. 378-396, 2015.

[12] S. Feng, X. Xinjilefu, C. G. Atkeson, and J. Kim, "Robust dynamic walking using online foot step optimization," in IEEE/RSJ Int. Conf. on Intelligent Robots and Systems (IROS), 2016.

[13] A. Sherikov, D. Dimitrov, and P.-B. Wieber, "Whole body motion controller with long-term balance constraints," in IEEE/RAS Int. Conf. on Humanoid Robotics (ICHR), 2016.

[14] S. Kuindersma, R. Deits, M. Fallon, A. Valenzuela, H. Dai, F. Permenter, T. Koolen, P. Marion, and R. Tedrake, "Optimization-based locomotion planning estimation and control design for the atlas humanoid robot," Autonomous Robots, vol. 40, pp. 429-455, 2015.

[15] J. Carpentier, S. Tonneau, M. Naveau, O. Stasse, and N. Mansard, "A versatile and efficient pattern generator for generalized legged locomotion," in IEEE/RAS Int. Conf. on Robotics and Automation (ICRA), 2016

[16] K. Bouyarmane, S. Caron, A. Escande, and A. Kheddar, Humanoid Robotics: a Reference. Spring, 2017, ch. Multi-Contact Motion Planning and Control.

[17] K. Kaneko, K. Harada, F. Kanehiro, G. Miyamori, and K. Akachi, "Humanoid robot hrp-3," in IEEE/RSJ Int. Conf. on Intelligent Robots and Systems (IROS), 2008, pp. 2471-2478.

[18] K. Kaneko, F. Kanehiro, M. Morisawa, K. Akachi, G. Miyamori, A. Hayashi, and N. Kanehira, "Humanoid robot hrp-4 - humanoid robotics platform with lightweight and slim body," in IEEE/RSJ Int. Conf. on Intelligent Robots and Systems (IROS), 2011, pp. 4400-4407.

[19] R. Tellez, F. Ferro, S. Garcia, E. Gomez, E. Jorge, D. Mora, D. Pinyol, J. Oliver, O. Torres, J. Velazquez, and D. Faconti, "Reem-b: An autonomous lightweight human-size humanoid robot," in IEEE/RAS Int. Conf. on Humanoid Robotics (ICHR), 2008, pp. 462-468.

[20] J. Saunders and V. I. et H. Eberhart, "The major determinants in normal and pathological gait," Journal of Bone Joint Surgery, vol. A, no. 135 , pp. 543-558, 1953.
[21] M. Focchi, G. Medrano-Cerda, T. Boaventura, M. Frigerio, C. Semini, J. Buchli, and D. G. Caldwell, "Robot impedance control and passivity analysis with inner torque and velocity feedback loops," Control Theory and Technology, vol. 14, no. 2, pp. 97-112, 2016.

[22] F. Negrello, M. Garabini, M. Catalano, J. Malzahn, D. Caldwell, A. Bicchi, and N. Tsagarakis, "A modular compliant actuator for emerging high performance and fall-resilient humanoids," in IEEE/RAS Int. Conf. on Humanoid Robotics (ICHR), 2015.

[23] J. Urata, J. Urata, T. Hirose, Y. Namiki, Y. Nakanishi, I. Mizuuchi, and M. Inaba, "Thermal control of electrical motors for high-power humanoid robots," in IEEE/RSJ Int. Conf. on Intelligent Robots and Systems (IROS), 2008.

[24] M. F. Fallon, P. Marion, R. Deits, T. Whelan, M. Antone, J. McDonald, and R. Tedrake, "Continuous humanoid locomotion over uneven terrain using stereo fusion." in IEEE/RAS Int. Conf. on Humanoid Robotics (ICHR), 2015

[25] N. Mansard, O. Stasse, P. Evrard, and A. Kheddar, "A versatile generalized inverted kinematics implementation for collaborative working humanoid robots: The stack of tasks," in International Conference on Advanced Robotics (ICAR), June 2009, p. 119.

[26] N. Ando, T. Suehiro, K. Kitagaki, T. Kotoku, and W.-K. Yoon, "Rtmiddleware: distributed component middleware for rt (robot technology)," in IEEE/RSJ Int. Conf. on Intelligent Robots and Systems (IROS), 2005

[27] M. Morisawa, K. Harada, S. Kajita, S. Nakaoka, K. Fujiwara, F. Kanehiro, K. Kaneko, and H. Hirukawa, "Experimentation of humanoid walking allowing immediate modification of foot place based on analytical solution," in IEEE/RAS Int. Conf. on Robotics and Automation (ICRA), 2007, pp. 3989-3994.

[28] M. Naveau, M. Kudruss, and O. Stasse, "A reactive walking pattern generator based on nonlinear model predictive control," IEEE/RAS Robotics and Automation Letters, 2016.

[29] J. Mirabel, S. Tonneau, P. Fernbach, A.-K. Seppälä, M. Campana, N. Mansard, and F. Lamiraux, "Hpp: a new software for constrained motion planning," in IEEE/RSJ Int. Conf. on Intelligent Robots and Systems (IROS), 2016 